Fragen zur GOÄ-Abrechnung
Kostenloser Leserservice!

Haben Sie Fragen zur privatärztlichen Abrechnung? Antworten gibt unser Experte Dr. med. Helmut Hoffmann, München.

Schreiben Sie an: Urban \& Vogel GmbH Redaktion HNO-NACHRICHTEN Aschauer Straße 30 81549 München Fax 0892030431400 E-Mail: gabriele.zoerrgiebel @springer.com

\title{
Muss eine private Zusatzversicherung für stationäre Behandlung prä- und postoperative Leistungen bezahlen, wenn diese vom operierenden Belegarzt erbracht und dem Patienten in Rechnung gestellt werden?
}

\begin{abstract}
Antwort: Belegärzte sind im Sinne des Gesetzes nicht am Krankenhaus angestellte Vertragsärzte, die berechtigt sind, ihre Patienten (Belegpatienten) im Krankenhaus unter Inanspruchnahme der hierfür bereitgestellten Dienste, Einrichtungen und Mittel stationär oder teilstationär zu behandeln, ohne hierfür vom Krankenhaus eine Vergütung zu erhalten. Sie sind per definitionem keine Krankenhausärzte.
\end{abstract}

Da die gesetzlichen und vertraglichen Regelungen der vor- und nachstationären Behandlung auf Krankenhäuser, Kliniken bzw. Krankenhausärzte und Klinikärzte abheben, gehört der Belegarzt nicht zu dem ärztlichen Personenkreis, der die vor- und nachstationäre Behandlung bei seinen GKV-Patienten, die er stationär aufgrund der vorhandenen Krankenzusatzversicherung als Privatpatienten behandelt hat, ebenfalls nach GOÄ abrechnen darf.

Gesetzlich versicherte Patienten mit privater Krankenzusatzversicherung für stationäre Behandlung sind für den Belegarzt bis zum Beginn der stationären Versorgung und nach Entlassung aus der stationären Behandlung Kassenpatienten. Die nicht stationär erbrachten Leistungen sind jeweils mit der KV abzurechnen.

Wenn ein Belegarzt seinen gesetzlich versicherten Patienten mit privater Krankenzusatzversicherung zur stationären Behandlung in ein anderes als sein Belegkrankenhaus einweist (z.B. Universitätsklinik, städt. Krankenhaus usw.) und der dort liquidationsberechtigte Krankenhausarzt ihn nach Beendigung der stationären Versorgung bittet, die nachstationäre Behandlung zu übernehmen, so muss der Belegarzt diese Leistungen unter Beachtung der zeitlichen Leistungsgrenze von 14 Tagen und der Häufigkeit von 7 Arzt-Patienten-Begegnungen direkt mit dem Krankenhaus abrechnen, in das er seinen Patienten eingewiesen hatte. Das Krankenhaus vergütet die nachstationäre Behandlung pauschal aus seinem DRG.
Empfehlenswert ist in diesen Fällen, vorab mit dem Krankenhaus eine vertragliche Regelung über die Höhe der Pauschalvergütung abhängig von der jeweiligen Diagnose zu treffen. Diese Regelung bedarf keiner Zustimmung der KV und auch keiner detaillierten GOÄ-Rechnung. In diesem Falle sind Pauschalen rechtens.

Der Patient, der bei einer PKV eine stationäre Krankenhauszusatzversicherung abgeschlossen hat, sollte vor Inanspruchnahme dieser Versicherung seinen Vertrag daraufhin überprüfen, ob die vor- und nachstationäre Behandlung durch den liquidationsberechtigten Krankenhausarzt in vollem Umfang erstattet wird.

Schlussendlich kann gesagt werden, dass die PKV (stationäre Krankenzusatzversicherung) ihrem Versicherungsnehmer die vom Belegarzt privatärztlich vor- und nachstationär berechneten Leistungen nicht erstatten muss, da es sich dabei um reine Kassenleistungen handelt. 